\title{
Einkommensverhältnisse der freien Ärzteschaft der Schweiz in den Jahren 1997/98 und im Jahr 2001
}

\author{
Vollerhebung*
}

N. Hasler

\begin{abstract}
* Gutachten erstellt im Auftrage der Verbindung der Schweizer Ärztinnen und Ärzte FMH. Das gesamte Gutachten mit 59 Seiten, 27 Tabellen und 28 Abbildungen kann im Internet über die Homepage der FMH www.fmh.ch über $\rightarrow$ Deutsch $\rightarrow$ Über uns $\rightarrow$ Ärzteeinkommen abgerufen werden. Die Numerierung der Tabellen und Abbildungen bezieht sich auf diesen vollständigen Bericht.
\end{abstract}

Korrespondenz:

Dr. med. Niklaus Hasler

Huzlenstrasse 3

CH-8604 Volketswil

E-Mail: nik@hin.ch

\section{A. Einleitung}

Mit Datum vom 13. November 2003 hat die Verbindung der Schweizer Ärztinnen und Ärzte den Auftrag erteilt, die Einkommensverhältnisse der freien Ärzteschaft der Schweiz für das Einkommensjahr 2001 erneut durch eine Vollerhebung $\mathrm{zu}$ analysieren.

Die Erhebung schliesst direkt an die früheren 19 Berichte an, welche von N. Hasler zwischen August 1977 und Mai 2002 erstellt wurden.

Hier folgt eine Zusammenfassung der wichtigsten Resultate, Tabellen und Abbildungen. In dieser Zusammenfassung werden bewusst nur die Zahlen für die Ärzte unter 66 Jahren angegeben. In der Vollversion sind jeweils auch die Zahlen für die Ärzte über 65 Jahre und die Zahlen für alle Ärzte (unabhängig vom Alter) angegeben.

Zudem werden in der Vollversion noch weitere Kriterien genau untersucht wie z.B. die Abhängigkeit des Einkommens von der Personallohnsumme, vom Alter des Arztes oder von der Zeit in Jahren seit der Praxiseröffnung.

Im nächsten Kapitel wird die wichtigste Änderung der Erhebung beschrieben, welche in den Zeitraum 1997/98 bis 2001 fällt, nämlich der Übergang von der Vergangenheitsbesteuerung auf die Gegenwartsbesteuerung.

\section{A.2. Übergang auf die Gegenwarts- besteuerung}

Im Rahmen der eidgenössischen Steuerharmonisierung wurde auch die Veranlagung der AHVBeiträge der Selbständigerwerbenden von der Vergangenheitsbesteuerung auf die Gegenwartsbesteuerung umgestellt.

Das Durchschnittseinkommen der Jahre 1997 und 1998 (30. Wehrsteuerperiode) ist die Grundlage für die Verfügung der in den Jahren 1999 und $2000 \mathrm{zu}$ bezahlenden persönlichen AHV-Beiträge.

Die Berufseinkommen der Jahre 1999 und 2000 fallen in die Bemessungslücke und können deshalb nicht erhoben und analysiert werden.
Ab dem Jahr 2001 gilt die Gegenwartsbesteuerung, d.h. das Einkommensjahr 2001 entspricht auch dem AHV-Beitragsjahr 2001.

Bis zu den Einkommen der 30. Wehrsteuerperiode (Einkommensjahre 1997/98) konnten die Ärzteeinkommen nur als zweijähriger Mittelwert im Zwei-Jahres-Rhythmus erhoben werden.

Ab dem Jahr 2001 können die Ärzteeinkommen aufgrund der verfügten persönlichen AHVBeiträge als Jahreseinkommen im Ein-JahresRhythmus erhoben werden.

\section{A.3. Probleme mit dem Übergang}

Der föderalistische Aufbau der Schweizerischen Eidgenossenschaft bedingt, dass einzelne Gesetze nicht zum gleichen Zeitpunkt in allen Kantonen der Schweiz realisiert werden. Beim Übergang auf die Gegenwartsbesteuerung zur Bemessung der persönlichen AHV-Beiträge der Selbständigerwerbenden haben die Kantone Tessin, Wallis und Waadt beschlossen, diesen Übergang erst auf den 1. Januar 2003 mit grosszügiger Genehmigung von Fristerstreckungen zur Abgabe der Unterlagen in Kraft zu setzen. Die übrigen Kantone haben den Wechsel bereits auf den 1. Januar 2001 vollzogen.

Aus diesem Grunde konnten die Wehrsteuerverwaltungen dieser drei Kantone für die Einkommen 2001 erst eine ganz geringe Anzahl von selbständigerwerbenden Ärzten prüfen und die geprüften Steuerzahlen der AHV-Ausgleichskasse Ärzte melden. Bei den Kantonen Tessin und Wallis sind es nur einige wenige Ärzte, weshalb die publizierten Durchschnittszahlen 2001 dieser Kantone zur Interpretation nicht verwendet werden dürfen. Für die Kantone Tessin und Wallis werden die definitiven Durchschnittszahlen 2001 voraussichtlich erst Mitte 2005 vorliegen. Beim Kanton Waadt sind bereits knapp die Hälfte aller Ärzte verfügt und behandelt worden, so dass die Zahlen Waadt 2001 weiterverwendet werden können. 


\section{Resultate}

\section{D.1. Allgemeines}

Zur Überprüfung der im Mai 2002 publizierten Einkommenszahlen 1997/98 wurde diese Werte bei der vorliegenden Erhebung neu analysiert. Die revidierten, jetzt publizierten Werte liegen durchwegs deutlich tiefer, aber doch recht nahe an den Werten der Erhebung vom Mai 2002:

- für alle Ärzte unabhängig vom Alter: (Mittelwert des AHV-pflichtigen Einkommens 198772 statt $207176=-4,1 \%$; Zentralwert des AHV-pflichtigen Einkommens 163600 statt $175700=-6,9 \%$;

- für alle Ärzte unter 66 Jahren: (Mittelwert des AHV-pflichtigen Einkommens 204624 statt $213508=-4,2 \%$; Zentralwert des AHV-pflichtigen Einkommens 169500 statt $181500=-6,6 \%)$;

- für alle Ärzte über 65 Jahre: (Mittelwert des AHV-pflichtigen Einkommens 85357 statt $89882=-5,0 \%$; Zentralwert des AHV-pflichtigen Einkommens 52300 statt $58900=$ $-11,2 \%)$.

Für die einzelnen Spezialitäten sind die Unterschiede äusserst uneinheitlich und unterschiedlich. Hier einige Beispiele:

- für die Gynäkologen unter 66 Jahren (Mittelwert des AHV-pflichtigen Einkommens 255095 statt $282964=-9,8 \%$, Zentralwert des AHV-pflichtigen Einkommens 222800 statt $239300=-6,9 \%$ );

- für die Urologen unter 66 Jahren (Mittelwert des AHV-pflichtigen Einkommens 341393 statt $341406=-0,0 \%$, Zentralwert des AHV-pflichtigen Einkommens 325200 statt $319300=+1,6 \%)$;

- für die Psychiater unter 66 Jahren (Mittelwert des AHV-pflichtigen Einkommens 118037 statt $124606=-5,3 \%$, Zentralwert des AHV-pflichtigen Einkommens 113100 statt $119200=-5,1 \%$;

- für die Allgemeinpraktiker unter 66 Jahren (Mittelwert des AHV-pflichtigen Einkommens 183420 statt $192004=-4,50 \%$, Zentralwert des AHV-pflichtigen Einkommens 170400 statt $179200=-4,9 \%$ ).

Genauso wie die absoluten Werte für die Einkommen 1997/98 müssen die Veränderungen der Einkommen zwischen 1995/96 und 1997/98 wie untenstehend angegeben korrigiert werden.

\section{Einkommensentwicklung 1995/96 bis $1997 / 98$}

Vergleich Gutachten Mai 2002 zu Gutachten Juli 2004.

\section{Teuerung 1995/96 bis 1997/98: +0,91\%}

Veränderung des mittleren AHV-pflichtigen

Ärzteeinkommens 1995/96 bis 1997/98

- alle Ärzte unabhängig vom Alter: $-2,15 \%$ statt $+1,99 \%$;

- Ärzte unter 66 Jahren: $-2,34 \%$ statt $+1,90 \%$;

- Ärzte über 65 Jahre: $-1,46 \%$ statt $+3,74 \%$.

Veränderung des zentralen AHV-pflichtigen

Ärzteeinkommens 1995/96 bis 1997/98

- alle Ärzte unabhängig vom Alter: $-1,62 \%$ statt $+5,65 \%$;

- Ärzte unter 66 Jahren: $-1,17 \%$ statt $+5,83 \%$;

- Ärzte über 65 Jahre: $-1,69 \%$ statt $+10,71 \%$.

\section{Einkommensentwicklung 1997/98 bis 2001}

Der Anstieg der Teuerung zwischen 1997/98 und 2001 betrug $+3,40 \%$. Im gleichen Zeitraum ist das mittlere Ärzteeinkommen (AHV-pflichtiges Berufseinkommen) für folgende Teilgesamtheiten der freien Ärzteschaft der Schweiz wie folgt angestiegen:

- alle Ärzte: von 198772 auf 205446 um $+3,36 \%$;

- Ärzte unter 66 Jahren: von 204624 auf $212494 \mathrm{um}+3,85 \%$;

- Ärzte über 65 Jahre: von 85357 auf 94152 um $+10,30 \%$.

Das zentrale Ärzteeinkommen (das Einkommen der einen Hälfte der angegebenen Ärzte liegt unter, das Einkommen der anderen Hälfte der angegebenen Ärzte liegt über dem zentralen Ärzteeinkommen) ist im gleichen Zeitraum für die gleichen Teilgesamtheiten wie folgt angestiegen:

- alle Ärzte: von 163600 auf 165200 um $+0,98 \%$;

- Ärzte unter 66 Jahren: von 169500 auf 172200 um $+1,59 \%$;

- Ärzte über 65 Jahre: von 53200 auf 58900 um $+10,71 \%$.

Mit Ausnahme der Ärzte über 65 Jahre hat sich das mittlere Ärzteeinkommen zwischen 1997/98 und 2001 genau gleich wie die Teuerung entwickelt. Es ist ein reales Ansteigen von 0,06\% bis $0,45 \%$ festzustellen. Beim zentralen Ärzteeinkommen hingegen ist ein reales Absinken von $1,81 \%$ bis $2,42 \% \mathrm{zu}$ verzeichnen. 
Bei den Ärzten über 65 Jahre ist beim mittleren Ärzteeinkommen ein realer Zuwachs von 6,90\% und beim mittleren Ärzteeinkommen ein realer Zuwachs von 7,31\% festzustellen.

Wenn die Zahlen zusammen mit den weiteren definitiv verfügten AHV-Einkommen in ein bis zwei Jahren erneut analysiert werden können und sich dabei um rund 2 bis 3 Prozent tiefere Werte ergeben, wird sich die teuerungsneutrale Entwicklung und der geringe reale Zuwachs mit Sicherheit in einen realen Einkommensverlust verwandeln.

Einkommensentwicklung 1971/72 bis 2001 Mit den revidierten Einkommenszahlen 1997/98 und den neuen Einkommenszahlen 2001 kann nun die Entwicklung des mittleren und des zentralen AHV-pflichtigen Einkommens der freien Ärzteschaft der Schweiz über gut drei Jahrzehnte verfolgt werden.
In den Abbildungen 13-16 (Anhang Seiten 52/53) sind die Indexreihen für das mittlere und das zentrale AHV-pflichtige Ärzteeinkommen (nur Ärzte unter 66 Jahren) sowohl nominal wie auch real (d.h. unter Berücksichtigung der Teuerung) dargestellt.

Von den vielen Tabellen und Abbildungen im Originalgutachten (einsehbar im Internet auf der Homepage der FMH www.fmh.ch) werden hier als Beispiele die Abbildungen 13 und 14 «Entwicklung des Mittelwertes des AHV-pflichtigen Berufseinkommens der freien Ärzteschaft der Schweiz unter 66 Jahren nominal und real» (unter Berücksichtigung der Teuerung) und die Tabellen 5 und 9 «Durchschnitt des AHV-pflichtiges Einkommens 1997/98 und 2001 der in freier Praxis tätigen unter 66 Jahre alten Ärzte nach Kantonen und nach Spezialitäten» gezeigt.

\section{Tabelle 23a}

Indexzahlen des AHV-pflichtigen Einkommens der freien Ärzteschaft der Schweiz (bis 1975/76 unter 63 Jahren, ab $1977 / 78$ unter 66 Jahren). 1971/72 bis 2001: Erster Teil 1971/72 bis 1985/86. Alle Indexreihen sind auf der Basis 1971/72=100,0 berechnet.

\begin{tabular}{|c|c|c|c|c|c|c|c|c|}
\hline & $1971 / 72$ & $1973 / 74$ & $1975 / 76$ & $1977 / 78$ & $1979 / 80$ & $1981 / 82$ & $1983 / 84$ & $1985 / 86$ \\
\hline Konsumentenpreise & 100,0 & 117,7 & 132,6 & 136,2 & 144,7 & 161,6 & 173,4 & 182,6 \\
\hline Veränderung & $+17,7 \%$ & $+12,7 \%$ & $+2,7 \%$ & $+6,2 \%$ & $+11,7 \%$ & $+7,3 \%$ & $+5,3 \%$ & \\
\hline AHV-pflichtiges Ärzteeinkommen: Mittelwert & 129340 & 149560 & 168250 & 158011 & 158048 & 164685 & 166601 & 168423 \\
\hline Veränderung & $+15,6 \%$ & $+12,5 \%$ & $-6,1 \%$ & $+0,0 \%$ & $+4,2 \%$ & $+1,2 \%$ & $+1,1 \%$ & \\
\hline Index nominal & 100,0 & 115,6 & 130,1 & 122,2 & 122,2 & 127,3 & 128,8 & 130,2 \\
\hline Index real & 100,0 & 98,2 & 98,1 & 89,7 & 84,5 & 78,8 & 74,3 & 71,3 \\
\hline AHV-pflichtiges Ärzteeinkommen: Zentralwert & 114950 & 132070 & 148000 & 142000 & 139800 & 144300 & 143700 & 143600 \\
\hline Veränderung & $+14,9 \%$ & $+12,1 \%$ & $-4,1 \%$ & $-1,5 \%$ & $+3,2 \%$ & $-0,4 \%$ & $-0,1 \%$ & \\
\hline Index nominal & 100,0 & 114,9 & 128,8 & 123,5 & 121,6 & 125,5 & 125,0 & 124,9 \\
\hline Index real & 100,0 & 97,6 & 97,1 & 90,7 & 84,0 & 77,7 & 72,1 & 68,4 \\
\hline \multicolumn{9}{|l|}{ Arbeiterlöhne } \\
\hline Index nominal & 100,0 & 125,8 & 144,2 & 151,0 & 162,6 & 183,9 & 200,1 & 212,8 \\
\hline Index real & 100,0 & 106,9 & 108,7 & 110,9 & 112,4 & 113,8 & 115,4 & 116,5 \\
\hline \multicolumn{9}{|l|}{ Angestelltenlöhne } \\
\hline Index nominal & 100,0 & 123,7 & 142,5 & 150,9 & 162,5 & 183,0 & 198,8 & 211,8 \\
\hline Index real & 100,0 & 105,1 & 107,5 & 110,8 & 112,3 & 113,2 & 114,6 & 116,0 \\
\hline
\end{tabular}


Tabelle 23b

Indexzahlen des AHV-pflichtigen Einkommens der freien Ärzteschaft der Schweiz (bis 1975/76 unter 63 Jahren, ab 1977/78 unter 66 Jahren). 1971/72 bis 2001: Zweiter Teil 1985/86 bis 2001. Alle Indexreihen sind auf der Basis 1971/72=100,0 berechnet.

\begin{tabular}{|c|c|c|c|c|c|c|c|c|}
\hline & $1985 / 86$ & $1987 / 88$ & $1989 / 90$ & 1991/92 & $1993 / 94$ & $1995 / 96$ & $1997 / 98$ & 2001 \\
\hline Konsumentenpreise & 182,6 & 187,7 & 200,7 & 222,4 & 235,3 & 241,6 & 243,8 & 252,1 \\
\hline Veränderung & $+2,8 \%$ & $+6,9 \%$ & $+10,8 \%$ & $+5,8 \%$ & $+2,7 \%$ & $+0,9 \%$ & $+3,4 \%$ & \\
\hline AHV-pflichtiges Ärzteeinkommen: Mittelwert & 168423 & 184599 & 189044 & 201577 & 209092 & 209529 & 204624 & 212494 \\
\hline Veränderung & $+9,6 \%$ & $+2,4 \%$ & $+6,6 \%$ & $+3,7 \%$ & $+0,2 \%$ & $-2,3 \%$ & $+3,8 \%$ & \\
\hline Index nominal & 130,2 & 142,7 & 146,2 & 155,9 & 161,7 & 162,0 & 158,2 & 164,3 \\
\hline Index real & 71,3 & 76,0 & 72,8 & 70,1 & 68,7 & 67,1 & 64,9 & 65,2 \\
\hline AHV-pflichtiges Ärzteeinkommen: Zentralwert & 143600 & 154500 & 155500 & 163900 & 170700 & 171500 & 169500 & 172200 \\
\hline Veränderung & $+7,6 \%$ & $+0,6 \%$ & $+5,4 \%$ & $+4,1 \%$ & $+0,5 \%$ & $-1,2 \%$ & $+1,6 \%$ & \\
\hline Index nominal & 124,9 & 134,4 & 135,3 & 142,6 & 148,5 & 149,2 & 147,5 & 149,8 \\
\hline Index real & 68,4 & 71,6 & 67,4 & 64,1 & 63,1 & 61,8 & 60,5 & 59,4 \\
\hline \multicolumn{9}{|l|}{ Arbeiterlöhne } \\
\hline Index nominal & 212,8 & 226,0 & 246,6 & 279,8 & 296,3 & 303,7 & 307,3 & 319,8 \\
\hline Index real & 116,5 & 120,4 & 122,9 & 125,8 & 125,9 & 125,7 & 126,1 & 126,9 \\
\hline \multicolumn{9}{|l|}{ Angestelltenlöhne } \\
\hline Index nominal & 211,8 & 223,9 & 242,0 & 269,8 & 285,1 & 293,1 & 298,2 & 312,6 \\
\hline Index real & 116,0 & 119,3 & 120,6 & 121,3 & 121,1 & 121,3 & 122,3 & 124,0 \\
\hline
\end{tabular}

\section{Abbildung 13}

Entwicklung des Mittelwertes des AHV-pflichtigen Einkommens der freien Ärzteschaft der Schweiz unter 66 Jahren (nominal) $1971 / 72$ bis 2001

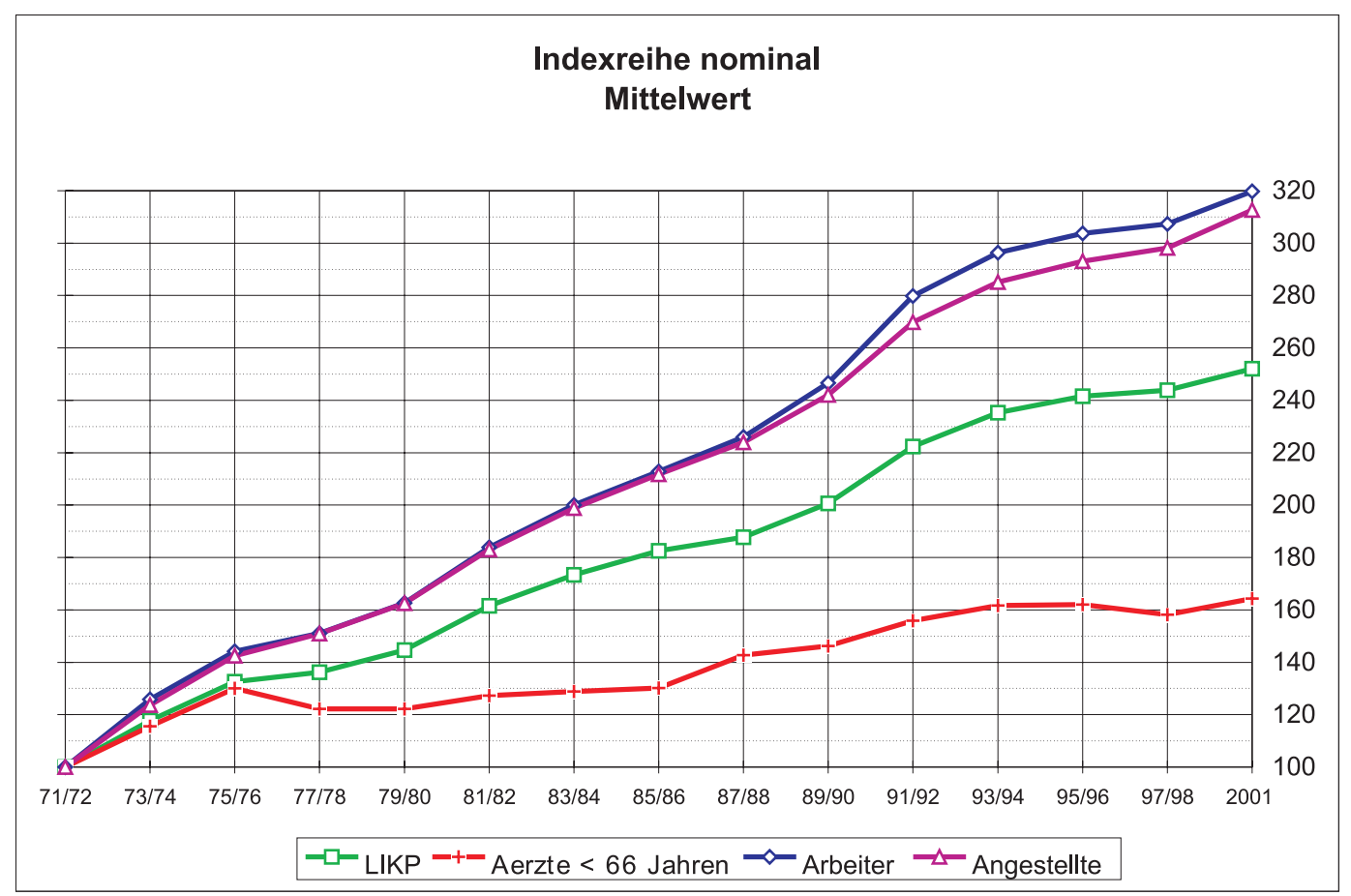


Abbildung 14

Entwicklung des Mittelwertes des AHV-pflichtigen Einkommens der freien Ärzteschaft der Schweiz unter 66 Jahren (real) $1971 / 72$ bis 2001 .

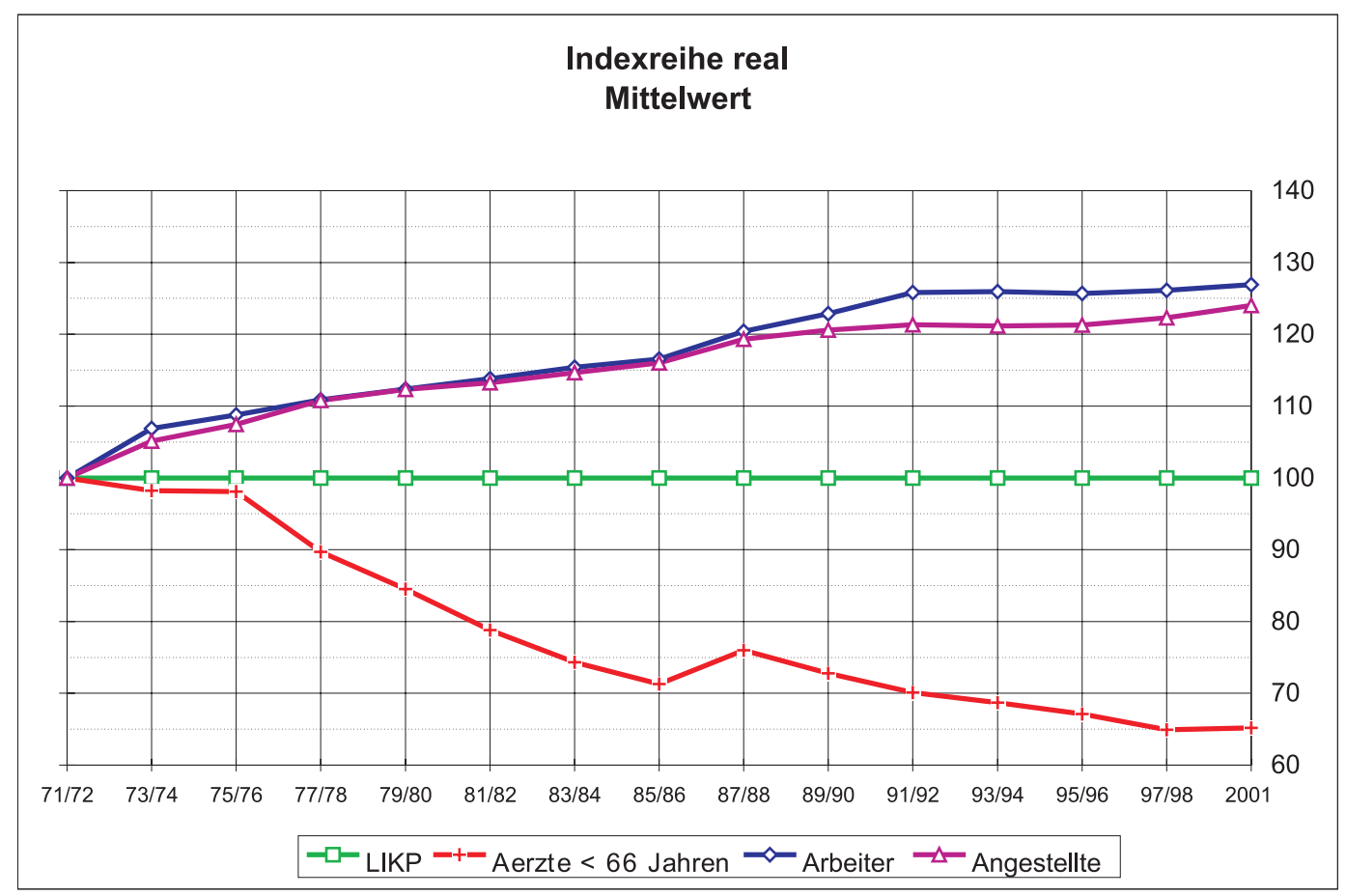

Tabelle 5

AHV-pflichtiges Einkommen aller in freier Praxis tätigen Ärzte, Durchschnitte nach Kantonen, Ärzte unter 66 Jahren. Wegen des Übergangs auf die Gegenwartsbesteuerung liegen keine Zahlen für die Kantone Tessin und Wallis vor.

\begin{tabular}{|c|c|c|c|c|c|c|c|}
\hline Kanton & Anzahl & $1997 / 98$ & Index & $+/-$ & Anzahl & 2001 & Index \\
\hline $\mathrm{ZH}$ & 2019 & 227236 & $111,1 \%$ & $+1,5 \%$ & 2129 & 230649 & $108,5 \%$ \\
\hline $\mathrm{BE}$ & 1506 & 209137 & $102,2 \%$ & $-1,7 \%$ & 1380 & 205685 & $96,8 \%$ \\
\hline LU & 396 & 208963 & $102,1 \%$ & $+4,8 \%$ & 288 & 218913 & $103,0 \%$ \\
\hline UR & 23 & 210613 & $102,9 \%$ & $-2,2 \%$ & 21 & 205905 & $96,9 \%$ \\
\hline$S Z$ & 115 & 249153 & $121,8 \%$ & $-10,3 \%$ & 14 & 223593 & $105,2 \%$ \\
\hline UW & 60 & 210493 & $102,9 \%$ & $+2,1 \%$ & 47 & 215013 & $101,2 \%$ \\
\hline $\mathrm{GL}$ & 42 & 198560 & $97,0 \%$ & $+2,6 \%$ & 40 & 203723 & $95,9 \%$ \\
\hline ZG & 130 & 221737 & $108,4 \%$ & $-5,3 \%$ & 46 & 209963 & $98,8 \%$ \\
\hline FR & 285 & 199655 & $97,6 \%$ & $+4,8 \%$ & 304 & 209203 & $98,5 \%$ \\
\hline SO & 343 & 190386 & $93,0 \%$ & $+6,8 \%$ & 319 & 203358 & $95,7 \%$ \\
\hline BS & 418 & 148520 & $72,6 \%$ & $+11,2 \%$ & 364 & 165136 & $77,7 \%$ \\
\hline $\mathrm{BL}$ & 464 & 206156 & $100,7 \%$ & $+13,8 \%$ & 460 & 234576 & $110,4 \%$ \\
\hline $\mathrm{SH}$ & 116 & 165286 & $80,8 \%$ & $+26,7 \%$ & 106 & 209386 & $98,5 \%$ \\
\hline AP & 85 & 191558 & $93,6 \%$ & $+22,4 \%$ & 68 & 234404 & $110,3 \%$ \\
\hline SG & 460 & 205341 & $100,4 \%$ & $+16,2 \%$ & 388 & 238504 & $112,2 \%$ \\
\hline GR & 274 & 178447 & $87,2 \%$ & $+15,0 \%$ & 221 & 205273 & $96,6 \%$ \\
\hline AG & 548 & 192337 & $94,0 \%$ & $-6,7 \%$ & 335 & 179419 & $84,4 \%$ \\
\hline TG & 277 & 234475 & $114,6 \%$ & $+3,6 \%$ & 249 & 243021 & $114,4 \%$ \\
\hline $\mathrm{TI}$ & 376 & 254018 & $124,1 \%$ & $-41,7 \%$ & & & \\
\hline VD & 852 & 198771 & $97,1 \%$ & $-2,9 \%$ & 411 & 193073 & $90,9 \%$ \\
\hline VS & 300 & 148478 & $72,6 \%$ & $-92,0 \%$ & & & \\
\hline NE & 135 & 139259 & $68,1 \%$ & $+5,2 \%$ & 140 & 146513 & $68,9 \%$ \\
\hline GE & 139 & 170178 & $83,2 \%$ & $-0,0 \%$ & 162 & 170149 & $80,1 \%$ \\
\hline JU & 75 & 139901 & $68,4 \%$ & $+0,9 \%$ & 74 & 141182 & $66,4 \%$ \\
\hline Schweiz & 9438 & 204624 & $100,0 \%$ & $+3,8 \%$ & 7571 & 212494 & $100,0 \%$ \\
\hline
\end{tabular}


Tabelle 9

AHV-pflichtiges Einkommen aller in freier Praxis tätigen Ärzte, Durchschnitte nach Spezialitäten, Ärzte unter 66 Jahren.

\begin{tabular}{|c|c|c|c|c|c|c|c|}
\hline Spezialität & Anzahl & $1997 / 98$ & Index & $+/-$ & Anzahl & 2001 & Index \\
\hline Anästhesie & 222 & 295186 & $144,3 \%$ & $-4,2 \%$ & 199 & 282774 & $133,1 \%$ \\
\hline Chirurgie & 447 & 260739 & $127,4 \%$ & $+3,5 \%$ & 350 & 269883 & $127,0 \%$ \\
\hline Dermatologie & 181 & 236273 & $115,5 \%$ & $+3,2 \%$ & 150 & 243931 & $114,8 \%$ \\
\hline Gynäkologie & 583 & 255095 & $124,7 \%$ & $-0,7 \%$ & 469 & 253434 & $119,3 \%$ \\
\hline Innere Medizin & 1838 & 208080 & $101,7 \%$ & $+4,7 \%$ & 1471 & 217873 & $102,5 \%$ \\
\hline Neurochirurgie & 24 & 350571 & $171,3 \%$ & $-4,1 \%$ & 13 & 336138 & $158,2 \%$ \\
\hline Neurologie & 122 & 178964 & $87,5 \%$ & $+1,8 \%$ & 92 & 182243 & $85,8 \%$ \\
\hline Ophthalmologie & 345 & 339977 & $166,1 \%$ & $+5,8 \%$ & 245 & 359674 & $169,3 \%$ \\
\hline Orthopädie & 201 & 277109 & $135,4 \%$ & $-1,0 \%$ & 181 & 274279 & $129,1 \%$ \\
\hline ORL & 181 & 267781 & $130,9 \%$ & $+9,9 \%$ & 131 & 294352 & $138,5 \%$ \\
\hline Pädiatrie & 459 & 178705 & $87,3 \%$ & $-2,3 \%$ & 353 & 174558 & $82,1 \%$ \\
\hline Physikalische Medizin & 138 & 191933 & $93,8 \%$ & $+15,7 \%$ & 111 & 222136 & $104,5 \%$ \\
\hline Psychiatrie & 1081 & 118037 & $57,7 \%$ & $-0,5 \%$ & 967 & 117389 & $55,2 \%$ \\
\hline Kinderpsychiatrie & 165 & 102938 & $50,3 \%$ & $-1,7 \%$ & 145 & 101235 & $47,6 \%$ \\
\hline Radiologie & 111 & 346514 & $169,3 \%$ & $+16,6 \%$ & 90 & 403877 & $190,1 \%$ \\
\hline Urologie & 76 & 341393 & $166,8 \%$ & $-3,6 \%$ & 72 & 329133 & $154,9 \%$ \\
\hline Allgemeinmedizin & 3250 & 183420 & $89,6 \%$ & $+8,0 \%$ & 2521 & 198139 & $93,2 \%$ \\
\hline Spezialisten & 6188 & 215761 & $105,4 \%$ & $+1,8 \%$ & 5050 & 219661 & $103,4 \%$ \\
\hline Allgemeinmediziner & 3250 & 183420 & $89,6 \%$ & $+8,0 \%$ & 2521 & 198139 & $93,2 \%$ \\
\hline Alle & 9438 & 204624 & $100,0 \%$ & $+3,8 \%$ & 7571 & 212494 & $100,0 \%$ \\
\hline
\end{tabular}

\section{F.8. Gesamtaufwand der Volkswirtschaft für die freie Ärzteschaft}

Es ist sehr schwierig, den Gesamtaufwand der Volkswirtschaft für die freie Ärzteschaft in der Schweiz zu erheben, da verschiedene Berechnungsfaktoren nur mit recht deutlicher Unsicherheit erhoben werden können, wie Unkostensatz der Arztpraxis oder die genaue Gesamtzahl der Ärzte in freier Praxis in der Schweiz.

Für die Jahre 1997 und 1998 liegt der volkswirtschaftliche Gesamtaufwand für die freie Ärzteschaft der Schweiz zwischen 7,099 und 8,352 Mia., was 1,82 bis $2,14 \%$ des Bruttosozialproduktes BSP der Schweiz ausmacht.

Wenn wir von der oberen möglichen Grenze von 8,352 Mia. ausgehen, entspricht dies 20,77\% der gesamten Ausgaben für das Gesundheitswesen in der Schweiz. Bei der angegebenen Untergrenze von 7,099 Mia. wären es 17,65\%.

Nach Angaben der Pharma Information benötigten die Spitäler im Jahre 1996 für die stationäre und ambulante Betreuung 21,279 Mia. oder $53,52 \%$ des Gesamtaufwandes für das Gesundheitswesen.

Gemäss Angaben der Pharma Information betragen die Gesamtaufwendungen für die freie Ärzteschaft (inkl. selbstdispensierter Medikamente) 7,017 Mia. oder 17,45\% des Gesamtaufwandes für das Gesundheitswesen.
Die Totaleinkommen aller Ärzte in freier Praxis belaufen sich dabei auf 2,698 Mia. oder $6,79 \%$ des Gesamtaufwandes für das Gesundheitswesen.

Für das Jahr 2001 liegt der volkswirtschaftliche Gesamtaufwand für die freie Ärzteschaft der Schweiz zwischen 8,516 und 10,103 Mia., was 2,01 bis $2,39 \%$ des Bruttosozialproduktes BSP der Schweiz ausmacht.

Wenn wir für das Jahr 2001 von der oberen möglichen Grenze von 10,103 Mia. ausgehen, entspricht dies 20,77\% der gesamten Ausgaben für das Gesundheitswesen in der Schweiz. Bei der angegebenen Untergrenze von 7,099 Mia. wären es für das Jahr 1998 17,65\%.

Nach Angaben der Pharma Information benötigten die Spitäler im Jahre 2001 für die stationäre und ambulante Betreuung 25,137 Mia. oder $54,49 \%$ des Gesamtaufwandes für das Gesundheitswesen.

Gemäss Angaben der Pharma Information betragen die Gesamtaufwendungen im Jahr 2001 für die freie Ärzteschaft (inkl. selbstdispensierter Medikamente) 7,560 Mia. oder 16,39\% des Gesamtaufwandes für das Gesundheitswesen.

Die Totaleinkommen aller Ärzte in freier Praxis belaufen sich dabei auf 2,981 Mia. oder $6,46 \%$ des Gesamtaufwandes für das Gesundheitswesen. 


\section{F.9. Tendenzen}

Die Ärzteeinkommen haben sich im Zeitraum 1997/98 bis 2001 im Gleichschritt mit der Steigerung des Lebenskostenindexes entwickelt. Es ist keine zusätzliche Steigerung der Ärzteeinkommen festzustellen.

Ganz generell kann festgestellt werden, dass in den letzten Jahren die Einkommen der operativ tätigen Ärzte etwas weniger rasch angestiegen sind als diejenigen der nicht-operativ tätigen Ärzte (= Grundversorger).
Es wird in den nächsten zwei bis vier Jahren interessant sein, die Auswirkungen des TARMEDTarifs zu beurteilen, der ja auch zum Ziel hat, die operativ-technischen Leistungen zugunsten der ärztlich-intellektuellen Leistungen abzuwerten.

Die Gegenwartsbesteuerung erlaubt nun, die Einkommensverhältnisse der freien Ärzteschaft der Schweiz in jährlichem und nicht nur in zweijährigem Rhythmus zu analysieren. 